\title{
Erratum: Pulmonary delivery of ISCOMATRIX influenza vaccine induces both systemic and mucosal immunity with antigen dose sparing
}

\section{JLJ Wee, J-PY Scheerlinck, KJ Snibson, S Edwards, M Pearse, C Quinn and P Sutton}

Mucosal Immunology (2008) 1, 489-496; doi:10.1038/mi.2008.59

For the above referenced article, there was a typesetting error made to the $x$ axis labels in Figure 2. The correct Figure 2 is shown below.
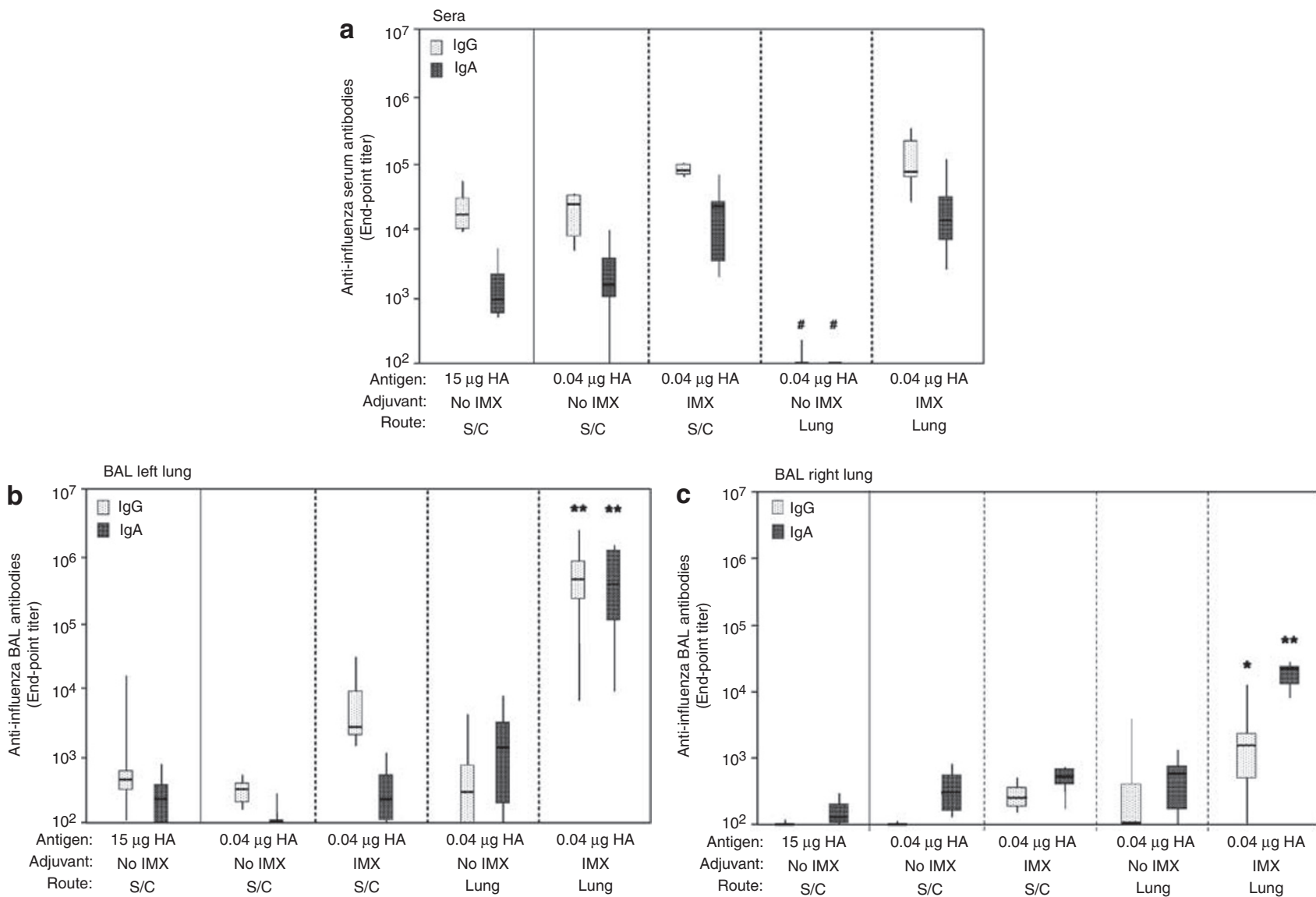

Figure 2 Induction of systemic and mucosal antibodies by immunization with extremely low doses of influenza antigen is dependent on both adjuvant and pulmonary delivery. In a single experiment, sheep $(\mathrm{n}=8)$ were immunized three times with $0.04 \mu \mathrm{g}$ influenza antigen (HA), with or without $100 \mu \mathrm{g}$ ISCOMATRIX adjuvant (IMX), delivered either via the lungs or subcutaneously $(\mathrm{S} / \mathrm{C})$. Positive controls $(\mathrm{n}=8)$ received $15 \mathrm{lg}$ antigen alone, S/C. Samples were collected after the third immunization, and IgA and IgG anti-influenza antibody titers determined by ELISA with pre-immunization antibody levels subtracted. (a) Serum antibody response; (b) lung washings (BAL) from the left lung where vaccine was delivered; (c) BAL from the contralateral right lung that was not directly exposed to antigen. Plots present the median titer (horizontal bar), interquartile ranges (boxed region) and 10th and 90th percentiles (error bars). For significance, data were log transformed and compared by ANOVA with Dunnett's post hoc analysis. There was no significant difference between titers in sheep vaccinated subcutaneously with $0.04 \mu \mathrm{g}$ antigen (with or without IMX adjuvant) and the positive controls. ${ }^{\#}$ Significantly lower antibody levels compared with all other groups $(P<0.001)$. *Significantly higher antibody levels compared with control group $(P<0.01) .{ }^{* *}$ Significantly higher antibody levels compared with all other groups $(P<0.02)$. 